Research Paper

\title{
Long-term Cultured Human Neural Stem Cells Undergo Spontaneous Transiormation to Tumor-Initiating Cells
}

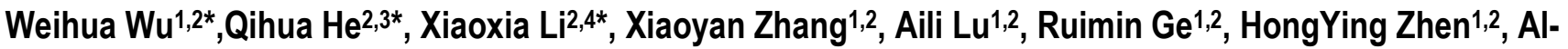 \\ fred E. Chang ${ }^{5}$, Qiao $\mathrm{Li}^{5 凶}$, Li Shen ${ }^{1,2 \bowtie}$
}

1. Department of Cell Biology, Peking University Health Science Center; Beijing 100191, China;

2. Peking University Stem Cell Research Center, Beijing 100191, China;

3. Peking University Medical and Health Analysis Center; Beijing 100191, China;

4. Department of Physiology, Peking University Health Science Center; Beijing 100191, China;

5. Department of Surgery, University of Michigan Medical Center, Ann Arbor, Michigan 48109-0666, USA

*Weihua Wu, Qihua He and Xiaoxia Li contributed equally to this work.

Corresponding author: Li Shen, Email: shenli@bjmu.edu.cn; and Qiao Li, Email: qiaoli@umich.edu

( ) Ivyspring International Publisher. This is an open-access article distributed under the terms of the Creative Commons License (http://creativecommons.org/ licenses/by-nc-nd/3.0/). Reproduction is permitted for personal, noncommercial use, provided that the article is in whole, unmodified, and properly cited.

Received: 2011.04.27; Accepted: 2011.07.04; Published: 2011.07.23

\begin{abstract}
In this report, we describe the spontaneous malignant transformation of long-term cultured human fetal striatum neural stem cells (hsNSCs, passage 17). After subcutaneous transplantation of long-term cultured hsNSCs into immunodeficient nude mice, 2 out of 15 mice formed xenografts which expressed neuroendocrine tumor markers $\mathrm{CgA}$ and NSE. T1 cells, a cell line that we derived from one of the two subcutaneous xenografts, have undergone continuous expansion in vitro. These T1 cells showed stem cell-like features and expressed neural stem cell markers nestin and CD133. The T1 cells were involved in abnormal karyotype, genomic instability and fast proliferation. Importantly, after long-term in vitro culture, the T1 cells did not result in subcutaneous xenografts, but induced intracranial tumor formation, indicating that they adjusted themselves to the intracranial microenvironment. We further found that the T1 cells exhibited an overexpressed level of EGFR, and the CD133 positive T1 cells showed a truncation mutation in the exons 2-7 of the EGFR (EGFRvIII) gene. These results suggest that continuous expansion of neural stem cells in culture may lead to malignant spontaneous transformation. This phenomenon may be functionally related to EGFR by EGFRvIII gene mutation.
\end{abstract}

Key words: tumorigenesis, malignant transformation, epidermal growth factor receptor (EGFR), xenograft

\section{Introduction}

Neural stem cells (NSCs) have the capability of self-renewal because at least one of the daughter cells maintains neural stem cell potential by asymmetric cell division. NSCs differentiate into three major neural cell types, neurons, oligodendrocytes and astrocytes [1]. However, the proliferation and self-renewal properties of NSCs may cause malignant transformation [2]. Although spontaneous malignant transformation was rarely observed, it has been suggested that the spontaneous transformation of adult stem cells, such as human mesenchymal stem cells (BM-hMSCs) and NSCs, can induce tumor formation [3-4]. The spontaneous transformation of murine neural precursor cells has been reported by Johnson 
[5] and Florian [6]. NSCs have been considered a promising tool for cell transplantation therapy and tissue engineering [7]. In order to obtain a sufficient amount of NSCs for clinical use, extensive in vitro expansion of NSCs is usually necessary. However, it remains a concern regarding the security of the NSCs. A recent report revealed that the fetal neural stem cells transplanted into a patient with a hereditary neurodegenerative disease have grown into noncancerous tumors in the brain and spinal cord [8]. Cancer stem cells have recently been identified as cancer-initiating cells for solid malignancies such as breast cancer [10] and pediatric brain tumors [11]. Thus, normal NSCs may be prone to genomic instability and malignant transformation, particularly during long-term culture in vitro. Due to genomic instability, accumulation of DNA damage, loss of regulation in cell cycle, and deregulation of epigenetic signature may occur.

To investigate whether normal NSCs have tumorigenic potential, we inoculated human fetal striatum neural stem cells (hsNSCs) [12] subcutaneously into immunodeficient nude mice. We observed a spontaneous transformation of hsNSCs after 17 passages in vitro. Furthermore, a T1 tumor cell line that was derived from the hsNSCs following subcutaneous xenograft tumor formation was established and characterized. We found that the tumorigenicity of long-term cultured T1 cells showed strong selectivity in the microenvironment, which associated with the amplification of epidermal growth factor receptor (EGFR) gene and a constitutively active mutant form of the receptor, EGFRvIII.

\section{MATERIALS AND METHODS}

\section{Culture and enrichment of human fetal striatum neural stem cells}

The human fetal striatum neural stem cells were isolated and characterized as previously described [12].

\section{In vivo tumorigenicity assays}

All animal experiments were performed in accordance with the guidelines of the Animal Care Committee, Peking University Health Science Center. Immunodeficient nude mice (4-5 weeks of age) were purchased from the Experimental Animal Department, Peking University Health Science Center. Experimental group with 15 mice was injected with hsNSCs at passage 17 and was examined for tumorigenicity. Other 5 and 10 mice were used as positive control (injected with Hela cells) and negative control (injected with saline solution), respectively. $3 \times 10^{6}$ cells in $300 \mu \mathrm{l}$ saline solution were subcutaneously injected into each mouse. The animals were monitored regularly for tumor occurrence throughout the whole experiment period.

\section{Isolation and culture of primary tumor cells from subcutaneous xenografts}

Immunodeficient nude mice with primary tumor formation were euthanized. The tumor cells were isolated, purified, and cultured in the primary culture medium of DMEM/F12 (Gibco, USA) supplemented with $10 \%$ fetal bovine serum (FBS, Hyclone, USA), EGF (20 ng/ml, PeproTech, USA), bFGF (20 ng/ml, PeproTech, USA), and B27 (50x, Invitrogen, USA). When adherent clones reached about $95 \%$ confluence, they were transferred to a new flask and cultured continuously to form a monolayer. The populations of tumor cells were isolated from neoplasias after at least five passages. We named this cell population as T1 cells.

\section{Serial subcutaneous xenografts}

The xenograft tissues were obtained through serial subcutaneous passage in immunodeficient nude mice. Small non-necrotic xenograft tissues were mechanically crumbled and passed through a $100-\mu \mathrm{m}$ cell sieve (Falcon). Then 4-week-old immunodeficient nude mice were implanted subcutaneously with tiny tissue fragments of the xenografts. The animals were monitored regularly for tumor occurrence throughout the whole survival period. Five mice were inoculated for each experiment. The tumor formation rate and the size of the neoplasias were recorded, and the experiment was repeated.

\section{G-banding Chromosome Analysis}

Cell chromosome analysis was performed using the conventional Giemsa staining. Karyotypic analysis was performed using an Olympus microscope (Optronic cooled CCD) and the dedicated cytoscan-karyotyping FISH \& CGH system (United Biotechnology USA corporation).

\section{DNA fingerprinting analysis using human short tandem repeat (STR) markers}

DNA fingerprinting analysis was performed using genomic DNA from NSCs (passage 9 and 17). The control cells and T1 cells were examined for human STR marker expression using the PowerPlex ${ }^{\circledR} 16$ System Kit (Promega Corp, Madison, WI) on an automated ABI 310 Genetic Analyzer (Applied Biosystems, Foster City, CA). 


\section{Measurement of cell proliferation}

For cell cycle analysis, DNA was labeled with 20 $\mu \mathrm{g} / \mathrm{ml}$ propidium iodine (Sigma, USA). The cell cycle was analyzed using FACScalibur flow cytometry with ModiFit software. For the PCNA analysis, nuclear antigen was stained with fluorescein (Fluorescein isothiocyanate, FITC) labeled with anti-PCNA antibody (Becton Dicksinson). Mouse FITC-IgG2a (Becton Dicksinson) was used as isotype control. Stained cells were analyzed using FACScalibur flow cytometry (Becton Dicksinson). The result was shown as the overlaid histograms of PCNA with its isotype control.

\section{Flow Cytometric Immunophenotypic Studies}

For membrane antigen staining, cells were trypsinized into single cell suspension in a total volume of $100 \mu 1 \mathrm{PBS} / \mathrm{BSA}$ and immediately stained for $15 \mathrm{~min}$ at room temperature in darkness with fluorescein (FITC) labeled antibodies, including anti-CD133, CD117, CXCR4 and EGFR. Mouse IgG2a-FITC or IgG1-FITC (Becton-Dickinson) was used as isotype control. Stained cells were analyzed by FACScalibur flow cytometry (Becton-Dickinson).

\section{Intracranial tumorigenic assays}

Ten immunodeficient nude mice were divided equally into the experimental group and control group. $5 \times 105$ isolated tumor cells were intracranially injected into five immunodeficient nude mice. When symptom of hydrocephalus was observed, the intact brains were removed and paraffin embedded for $\mathrm{H} \& \mathrm{E}$ staining. Normal human fetal striatum neural stem cells were injected into the control mice. All surgical interventions and animal care were provided in accordance with the Guide for the Care and Use of Laboratory Animals of the National Institutes of Health.

\section{Immunofluorescence assays}

To stain the cytoplasmic and nuclear proteins, cells were incubated with relevant primary antibodies at $4^{\circ} \mathrm{C}$ overnight and subsequently with appropriate secondary fluorescence-conjugated antibodies. Mouse or rabbit normal serum was used as a negative control. The primary antibodies included a mouse anti-human antibody specific for nuclei (Chemicon, 1:100), a mouse anti-human antibody specific for the epidermal growth factor receptor (Chemicon, 1:100), and a rabbit anti-GFAP polyclonal antibody (Chemicon, 1:100). DAPI was used for nuclear staining. Fluorescence signals were detected using a Confocal microscope (Leica TCS SP5, German) or an Olympus immuno-fluorescent microscope (Model BX51TR) with an Apogee Instruments Microscopy Fluorescence System (Model KX85).

\section{Purification of CD133+ cells}

CD133+ cells were purified by magnetic cell sorting (MACS) using the CD133 MicroBead Kit (Miltenyi Biotech), and analyzed by the flow cytometry system using CD133/2-PE (Miltenyi Biotech). For reverse-transcriptase-PCR (RT-PCR) assay, total RNA was extracted from the sorted CD133+ cells. Complementary DNA (cDNA) was synthesized. 5' (sense) primer was: 5'-CTTCGGGGAGCAGCGATGCG AC-3', 3' (antisense) primer was: 5'ACCAATACCTATTCCGTTACAC-3'. RT-PCR products yielded two fragments, a 1044-bp (wild-type) fragment and a 243-bp (deletion product) fragment. PCR products were subcloned into the pGEM-T easy cloning vector (Invitrogen) and sequenced.

\section{RESULTS}

\section{Transformation of Long-term Cultured hsNSCs as Revealed in Subcutaneous Xenograft}

Among the 15 mice transplanted with cultured hsNSCs (passage 17), xenografts formed in two mice 3 months after implantation (Fig. 1A, 1B), whereas tumors formed in five of five mice transplanted with Hela cells. No tumor was formed in the negative control mice injected with saline (data not shown). These results suggested a risk of tumorigenesis from the transplantation of long-term cultured hsNSCs. The H\&E staining results showed that the neoplasias proliferated actively and were of heterogeneity of nucleus (Fig. 1E,1F). The immunohistochemistry results indicated that, pathologically, these tumors belonged to the neuroblastoma-like tissue pattern (Fig. 1G-1L). They were positive for CgA (Fig. 1G) and NSE (Fig. 1H), but were negative for CD99 (Fig. 1I), cytokeratin AE1/ AE3 (Fig. 1J), synaptophysin (Fig. 1K) and GFAP (Fig. 1L). The tumor cells form the hsNSC injected mice was isolated, purified and cultured, we named them as T1 cells. The Karyotype analyses were performed on passage $17 \mathrm{hsNSCs}$ and the T1 cells. Metaphases were determined for each karyotype analysis, and the representative karyotype was shown in Fig 1C and 1D. The chromosome dispersion of the T1 cells ranged from 60 to 70 chromosomes. A large number of different numerical and structural chromosomal aberrations were detected in the T1 cells but not in the parent hsNSCs, which showed a normal complement of chromosomes and no genetic abnormality (Fig. 1C, 1D).

\section{Establishment and characterization of the T1 cell line}

T1 cells were isolated as described in the Materials and Methods. To evaluate the proliferation ca- 
pacity of the T1 cells, the cell cycle was analyzed by flow cytometry. The percentages were $42.60 \%$ for the $\mathrm{S}$ phase cells (Fig. 2A), and $91.46 \%$ positive for the proliferation cell nuclear antigen (PCNA) (Fig. 2B). By the end of a period of at least five passages, the two types of cells exhibited the homogeneous morphology. At passage 5, the T1 cell line was purified, and mouse cell contamination was not detected using an anti-human nucleus antibody (Fig. 2C). A short tandem repeat (STR) analysis, which was conducted by the Center of Forensic Sciences Beijing Genomics Institute, was employed to monitor the genomic instability. STR loci consisted of short, repetitive sequence elements with 3 to 7 base pairs in length. The STR markers were employed to detect the variability between the T1 cells and the hsNSCs. The boxed numbers and corresponding peaks between the hsNSCs and the $\mathrm{T} 1$ cells were different (Fig. 2D-2F). These alterations indicated that the $\mathrm{T} 1$ cell line exhibited genomic instability. Based on these data, the T1 cells were confirmed as a pure population of tumor cells that originated from the transformation of the hsNSCs.

\section{Immunophenotype analysis of parental neural stem cells (hsNSCs) and T1 cells by FACS}

Variable levels of antigen expression were observed for the markers of cell's capacity of division and proliferation in the study, as shown in Fig 3. PCNA analysis results showed that T1 cells had a proliferative capacity that was stronger than the hsNSCs (Fig. 3A, B). Upon staining hsNSCs and T1 cells simultaneously for CD117, CXCR4, we observed a marked upregulated expression in T1 cells (Fig. 3C-F). In contrast, the expression of CD133 in hsNSCs and $\mathrm{T} 1$ cells was relatively uniform (Fig. 3G, 3H). Together, these data had revealed certain important molecular and biological changes that could contribute to the tumorigenicity of T1 cell line.

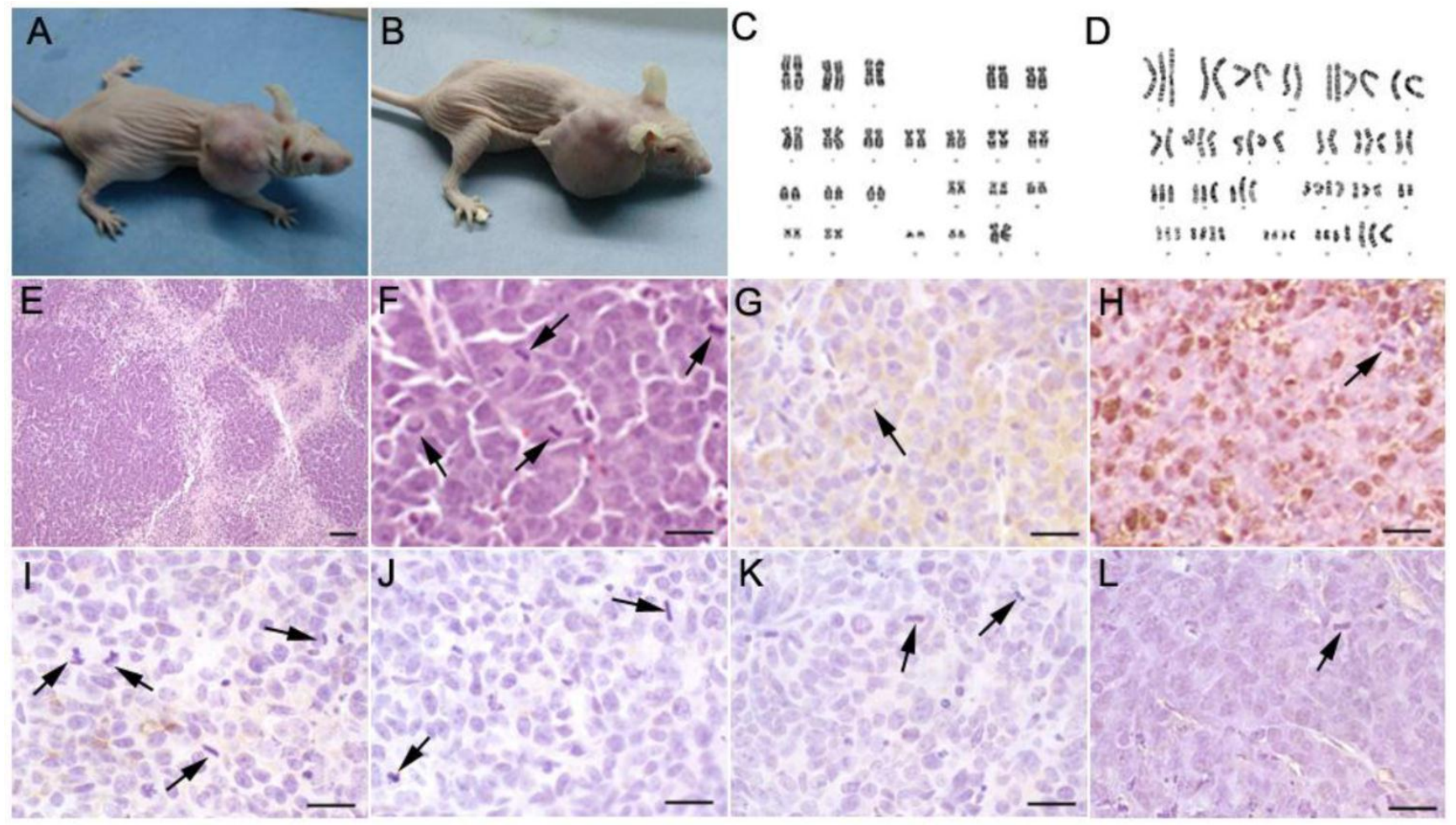

Fig. 1. Subcutaneous tumorigenesis in immunodeficient nude mice injected with long-term cultured hsNSCs (A, B). Tumors were observed in 2 out of 15 mice. Karyotype analyses were performed on passage 17 hsNSCs and T1 cells. The karyotype of hsNSCs was analyzed using the G-banding staining method. Normal human diploid karyotype was maintained throughout the in vitro culture period $(C)$, whereas a large number of different numerical and structural chromosomal aberrations were detected in the T1 cells (D). H\&E staining (E, F) showed that the neoplasias proliferated actively. Large areas of necrosis surrounded by highly packed tumor cells were observed within the tumors. Many mitotic figures were demonstrated. Immunohistochemistry results indicated that the isolated tumors were immunoreactive for neuroendocrine tumor markers $\mathrm{CgA}$ $(\mathrm{G})$ and NSE $(\mathrm{H})$, but were negative for CD99 (I), cytokeratin AE1/AE3 (J), synaptophysin (K) and GFAP (L). Black arrowheads show the mitosis of spindles or equatorial planes. Scale bar: E, $100 \mu \mathrm{m} ; \mathrm{F}-\mathrm{L}, 20 \mu \mathrm{m}$. 

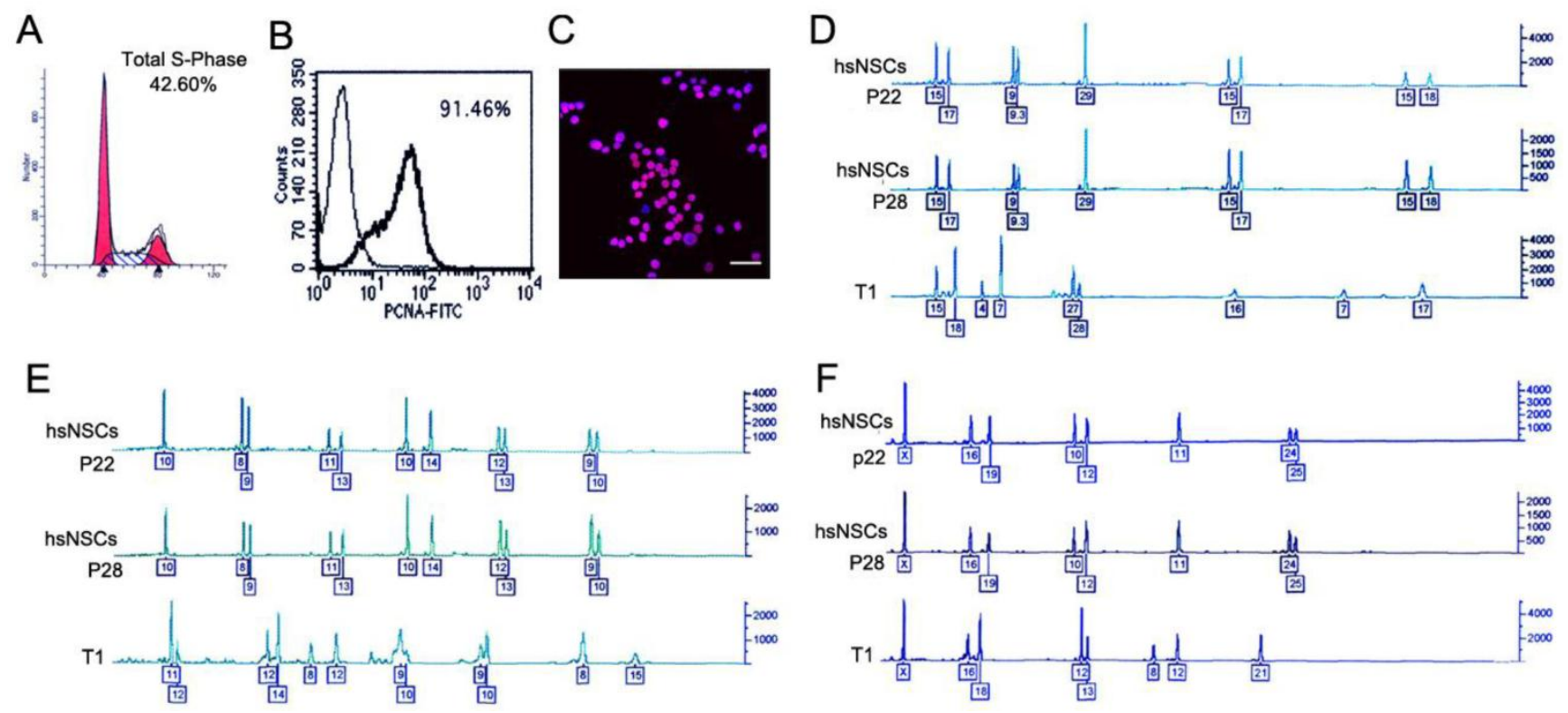

Fig. 2. Identification and characterization of T1 cell line. Cell cycle and PCNA analysis indicated that the percentages were 42.60\% (A) for the $S$ phase cells and $91.46 \%$ positive for PCNA (B) respectively in T1 cells. Immunofluorescence assay showed the image of nuclear staining, using an anti-human nucleus antibody (C). Nuclei were stained with DAPI (Scale bar: $100 \mu \mathrm{m})$. DNA fingerprinting with the STR markers of the donor hsNSCs (passage 22, 28 respectively) and the T1 cells was examined and compared (D-F). The boxed numbers and corresponding peaks of the hsNSCs at passage 22 and 28 were completely identical, but were significantly different from those of the T1 cells.

\section{The neoplasias of long-term cultured T1 cells were restricted in the brain after transplantation into nude mice}

To determine the in vivo tumorigenicity, the xenograft tissues were obtained through serial subcutaneous passage in immunodeficient nude mice according to the method described in the Material and Methods section. Serial subcutaneous transplantations of T1 xenograft tissues were performed and measured in the immunodeficient nude mice (Fig. 4A). However, we were surprised that during long-term in vitro culture, the T1 cells did not result in xenografts subcutaneously. Since the T1 cells were transformed from neural stem cells, we injected the T1 cells that were cultured in vitro for long periods of time into the brains of immunodeficient nude mice to test intracranial neoplasias. After one to two months, all five nude mice were fleshless and unresponsive. Histologically, the intracranial neoplasias were poorly differentiated tumors (Fig. 4B, 4C). These tumor cells invaded the surrounding normal nervous tissues. H\&E staining analysis revealed that these tumor cells had large, oval nuclei with some prominent nucleoli. Spindles or equatorial planes were also observed in some tumor cells, implying that these tumor cells could proliferate quickly (Fig. 4B, 4C). Immunofluorescence analysis indicated that the GFAP of the intracranial neoplasias was changed from the negative to positive in the subcutaneous xenografts (Fig. 4D-4F). H\&E staining (Fig 4G, 4H) show the sections of nude mice brain with hsNSC transplantation. Black arrow indicates the neural scar after neural stem cells transplantation. White arrow indicates the needle trajectory of neural stem cells transplantation (Scale bar: $1000 \mu \mathrm{m})$. These data indicated that the T1 cells could grow into tumors in the brain, and the neoplasias were similar to tumors derived from the nervous system. 

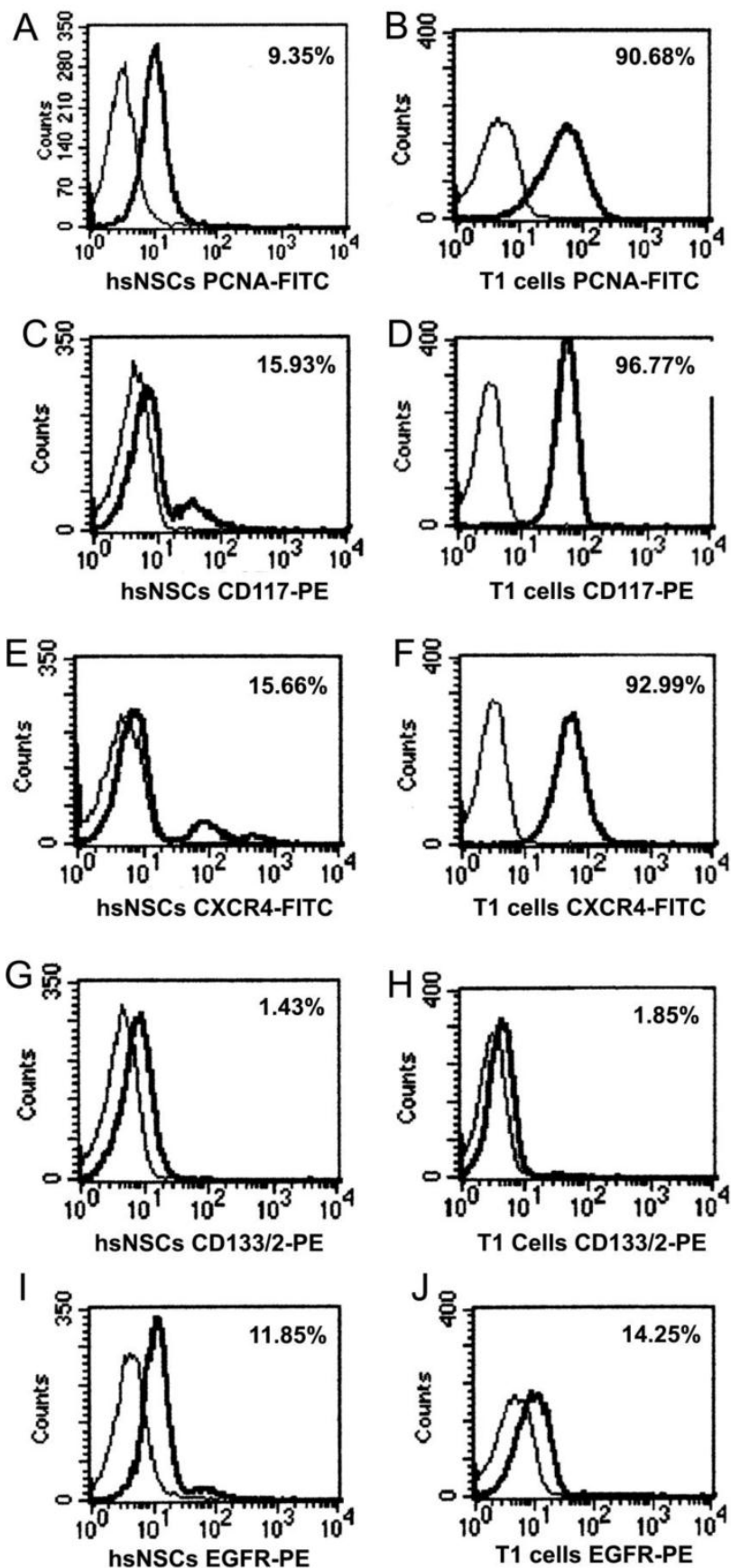

Fig. 3. Immunophenotype analysis of parental neural stem cells (hsNSCs) and T1 cells by FACS. PCNA analysis indicated that $\mathrm{T} 1$ cells had a stronger proliferative capacity than the parental neural stem cells (hsNSCs) (A-B). CXCR4 and CD117 were marked upregulated in T1 cells in comparison with hsNSCs (C-F). However, the expression of CD133 and EGFR in hsNSCs and T1 cells demonstrated no significant difference (G-J).

\section{T1 cell line shows stem cell-like features and initiates intracranial tumor formation}

From the results demonstrated above, the $\mathrm{T} 1$ cells exhibited strong proliferation activities. We further found that these T1 cells showed stem cell-like features and expressed neural stem cell markers nestin and CD133 (Fig. 5A). The environmental factors involved in the $\mathrm{T} 1$ cell line were also determined. Both CXCR4 (Fig. 5B) and EGFR (Fig. 5C) were detected during brain tumorigenesis. EGFRvIII was also identified in the T1 cells (Fig. 5D). CXCR4 in the T1 cells showed polarity (Fig. 5B), indicating that the T1 cells possessed the potential for migration under the action of chemokines. The sections of tumor tissues derived from $\mathrm{T} 1$ cell intracranial transplantation also expressed CXCR4, EGFR and EGFRvIII (Fig. 5E-5G). The glial cell marker GFAP was still positive (Fig. 5G). It is known that the mutant of the EGFR gene EGFRvIII was involved in the majority of cases of glioblastoma multiforme (GBMs) with EGFR amplification [13]. Thus, we separated CD133(+) and CD133(-) cells from the intracranial $\mathrm{T} 1$ tumors using the CD133-specific antibody (Fig.5I), and then determined the status of the EGFRvIII mutant. The RT-PCR assay was used to analyze the EGFRvIII mutation. We found that the CD133(-) T1 cells showed both the EGFR (1044-bp) and EGFRvIII (243-bp) bands, while the CD133(+) T1 cells only showed the EGFRvIII band (Fig. $5 \mathrm{H}$ ). The sequencing result of the EGFRvIII gene was shown in Fig.5J. Together, these data suggest that EGFRvIII played an important role in the brain tumor formation.

\section{DISCUSSION}

Adult human neural stem cells have been considered as a resource for cell therapy and tissue engineering [7, 13-14]. Due to their capabilities of self-renewal and differentiation towards the neural cells, NSCs provide an attractive tool for transplantation-based therapy of neurodegenerative diseases [15-16]. In vitro NSC expansion makes it possible to obtain sufficient amounts of NSCs for clinical use. However, a potential risk of NSC treatment might arise in that NSCs can paradoxically promote tumorigenesis. It has been reported that after prolonged in vitro culture, human adipose derived MSCs, murine bone marrow derived stem cells, and NSCs can become tumorigenic [3-6]. Long-term in vitro cultured NSCs may be particularly prone to genomic instability. Therefore, it is critical to evaluate the risk of human NSCs transformation after long-term in vitro culture.

In this study, following long-term hsNSC culture in vitro, we observed spontaneous malignant transformation following the subcutaneous transplantation of hsNSCs into immunodeficient nude mice. We established a T1 cell line derived from the tumor growing out of the transformed hsNSCs at passage 17 following subcutaneous xenograft. The T1 cell line 
showed stem cell-like features and expressed neural stem cell markers nestin and CD133. STR analysis indicated critical genomic instability that might have led to the transformation of hsNSCs. Thus, the T1 cells were involved in the abnormal karyotypes and genomic instability. Genetic alteration such as amplifications, mutations or deletions of genes might lead to a decoupling of the biological mechanisms involved in the regulation of normal cell growth and differentiation [11,17-20]. A difference between serial subcutaneous and intracranial tumorigenesis was observed in this study. During long-term in vitro culture, the T1 cells did not result in subcutaneous xenograft, but led to obvious intracranial tumor formation, indicating that these cells adjusted themselves to the intracranial microenvironment. The neoplasias were restricted in the brain and they opted for selecting their favorite microenvironment. Although it remains largely unknown why the transformations of the hsNSCs and T1 cells occurred in response to distinct microenvironments, it has been widely accepted that the host microenvironment plays a crucial role in cancer initiation and progression [21-23].

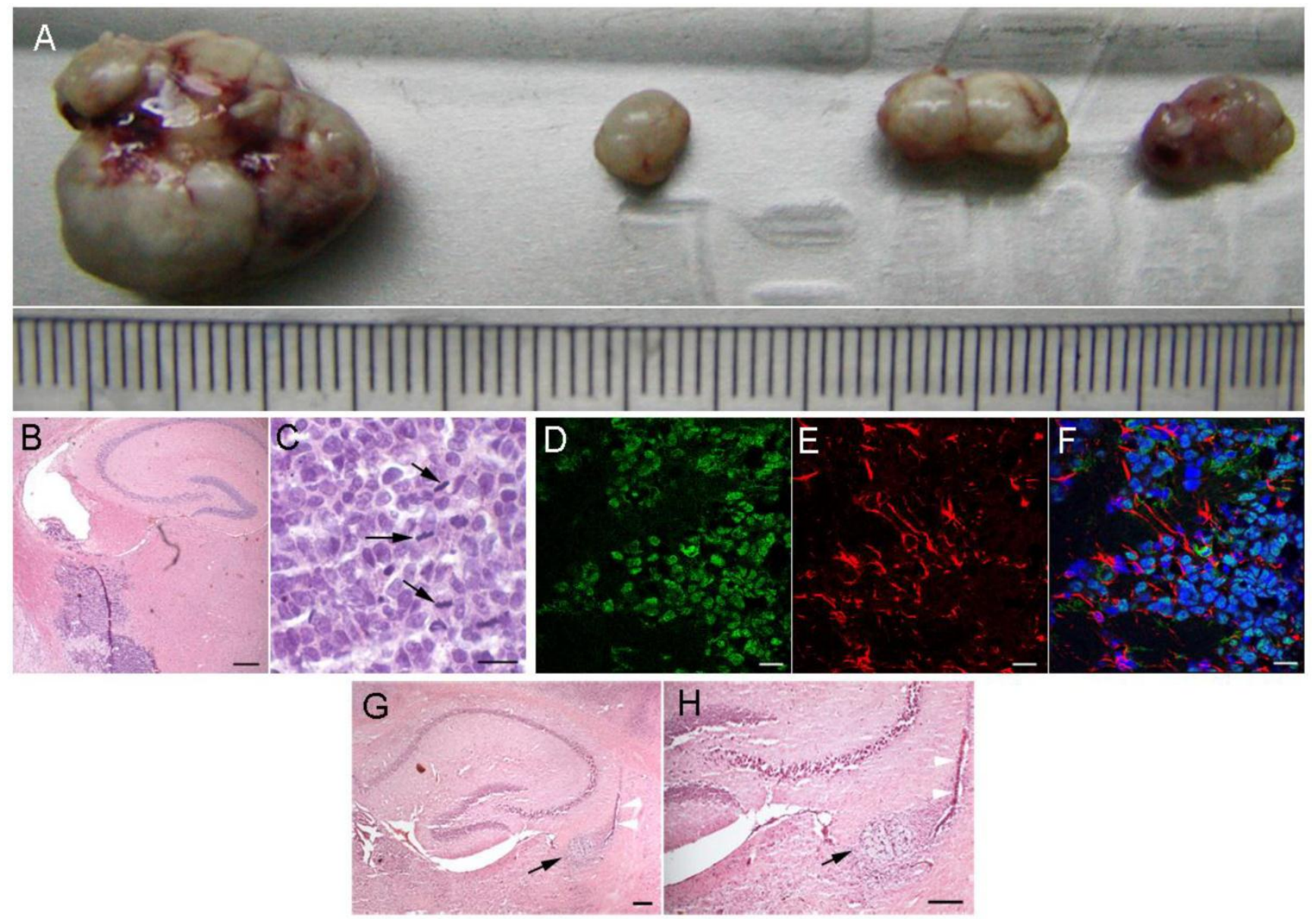

Fig. 4. Intracranial tumorigenicity of T1 cells in immunodeficient nude mice. Serial 4 subcutaneous passages of T1 tumor formation were shown $(\mathrm{A})$. The size of the tumors could be seen using the scale of the ruler, each grid represents $1 \mathrm{~mm}$. H\&E staining of the brain tissues of immunodeficient nude mice after T1 cell intracranial transplantation: space-occupying lesions in the lateral ventricle, as well as many atypical and prominent nuclei, were shown in high magnification view fields (Scale bar: B $100 \mu \mathrm{m} ; C 50 \mu \mathrm{m}$ ). Black arrowheads showed the mitosis of the equatorial plane (B, C). The brain tissue sections of immunodeficient nude mice after T1 cell transplantation were used for immunofluorescence analysis. The anti-human nucleus antibody staining was positive for the tumor cells (D). Some tumor cells were immunoreactive for glial cell's marker GFAP (E, F). Nuclei were detected using the DAPI staining showed in merged image (F) (Scale bar: 40 $\mathrm{mm}$ ). H\&E staining (G-H) show the sections of nude mice brain with hsNSC transplantation. Black arrow indicates the neural scar after neural stem cells transplantation. White arrow indicates the needle trajectory of neural stem cells transplantation (Scale bar: $1000 \mu \mathrm{m})$. 

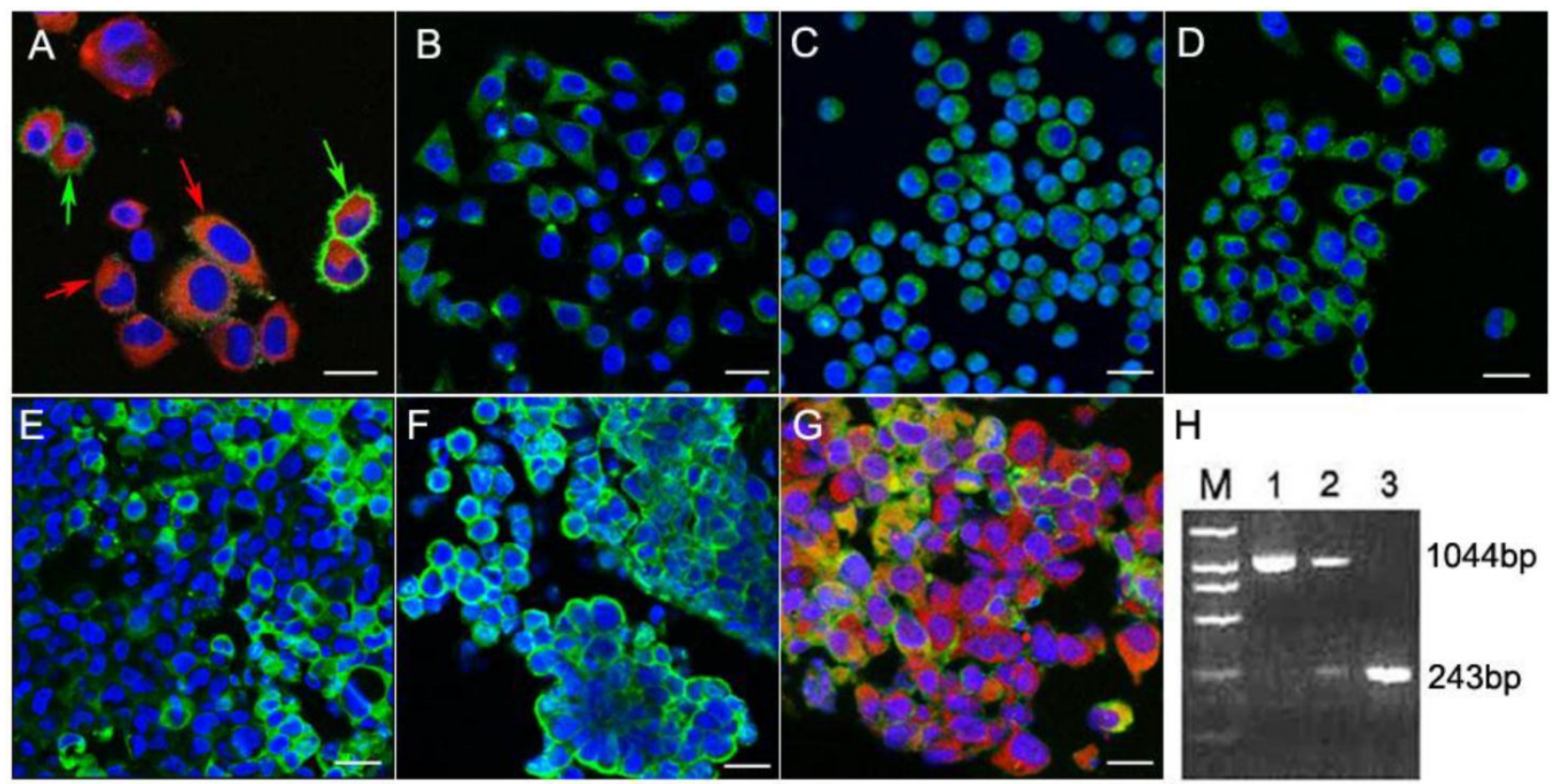

I

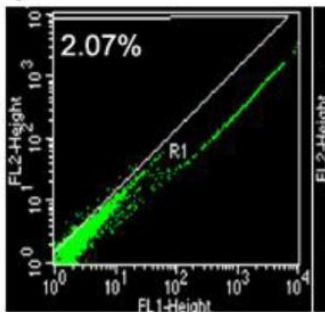

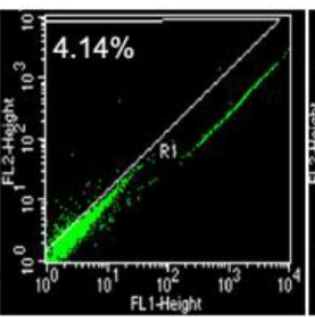

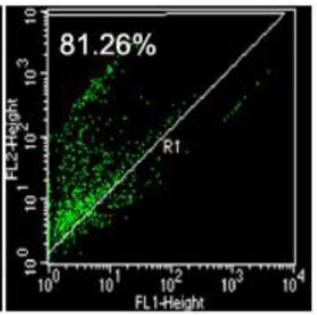

$\mathrm{J}$

Fig. 5. Immunofluorescence assays showed that the T1 cells expressed neural stem cell markers nestin (red \& red arrowheads) and CD133 (green \& green arrowheads A). Scale bar, $20 \mu \mathrm{m}$. CXCR4 (B), EGFR (C) and EGFRvIII (D) were expressed in the T1 cells. Scale bar, $50 \mu \mathrm{m}$ ). Immunoflurescence analyses of tumor tissue sections (Scale bar: $20 \mu \mathrm{m}$ ) derived from T1 cell intracranial transplantation are shown (E-G). EGFR (green) and GFAP (red) were both expressed by transplanted T1 cells (G). Nuclei were detected using the DAPI staining method (blue). The RT-PCR results showed that the products of EGFR and EGFRvIII were 1044-bp and 243-bp $(\mathrm{H})$, respectively. Lane 1 was for the neurospheres samples, showing EGFR 1044bp band; Lane 2 was for the CD133(-) T1 cells, showing two bands including EGFR (1044bp) and EGFRvIll (243bp); Lane 3 was for the CD133(+) T1 cells, and showed the 243-bp EGFRvlll band only. The CD133(+) and CD133(-) cells from intracranial T1 cell-induced tumor were separated using the CD133-specific antibody (I). Sequencing result confirmed the expression of the EGFRvIll gene $(\mathrm{J})$.

We detected strongly CD117 (KIT) expression in T1 cells using FACS. A growing body of evidence suggests that the KIT receptor involved in SCF signaling is highly activated, which enhances endothelial cell proliferation in the brain microvasculature both in vivo and in vitro [24-25]. Both CXCR4 and EGFR are critical receptors involved in biological pathways because of their roles in inflammation, development and tumorigenesis [26-28]. CXCR4 is an important molecule involved in the spread and progression of a variety of tumors [26,29-31]. Recent investigations have revealed that chemokines are often up-regulated within areas of brain pathology and may provide crucial signaling cues for stem cell migration $[23,32]$. Overexpression or activation of the epidermal growth factor receptor (EGFR), a receptor tyrosine kinase associated with cell proliferation and survival, is often found in tumors[33]. Crosstalk between EGFR and CXCR4 has recently been found to play an important role in the development of tumors [34]. Our findings revealed that $\mathrm{T} 1$ cells overexpress EGFR, and the CD133 positive $\mathrm{T} 1$ cells show a truncation mutation in the exons 2-7 of the EGFR gene (EGFRvIII). EGFRvIII is caused by deletion of exons 2 to 7 , resulting in a protein that lacks a ligand-binding domain and is constitutively activated. EGFRvIII is further resistant to down-regulation due to a low rate of receptor endocytosis [35-37]. Mutant forms of the EGFR gene have been reported to participate in tumorigenesis for patients with malignant gliomas [38-40]. 
In conclusion, our results strongly suggest that the risk of malignant spontaneous transformation might result from the genomic instability caused by the continuous expansion of neural stem cells in vitro. The microenvironments play a crucial role in the intracranial tumor initiation and progression. Since the mutant EGFR gene EGFRvIII was involved in the majority of cases of glioblastoma multiforme with EGFR amplification [13], the intracranial tumor formation observed in our study might be related to the EGFRvIII gene during the process of tumorigenesis.

\section{Acknowledgment}

This work was supported by the National Natural Science Foundation of China (grant 30471638) and the Beijing Natural Science Foundation (grant 7062041). This work was also supported by the Foundation of National Science and Technology Major Project Focusing on Drug Innovation from The Ministry of Science and Technology of the People's Republic of China and The National Basic Research Program of China 973 Project (grant 2011CB510200 and 2011CB707700). This work was partially supported by the Gillson Longenbaugh Foundation.

\section{Conflict of Interests}

The authors have declared that no conflict of interest exists.

\section{References}

1. Lendahl U, Zimmerman LB, McKay RD. CNS stem cells express a new class of intermediate filament protein. Cell 1990; 60: 585-595.

2. Vescovi AL, Galli R, Reynolds BA. Brain tumour stem cells. Nat Rev Cancer 2006; 6: 425-436.

3. Røsland GV, Svendsen A, Torsvik A, et al. Long-term cultures of bone marrow-derived human mesenchymal stem cells frequently undergo spontaneous malignant transformation. Cancer Res. 2009; 69: 5331-5339.

4. Casalbore P, Budoni M, Ricci-Vitiani L, et al. Tumorigenic potential of olfactory bulb-derived human adult neural stem cells associates with activation of TERT and NOTCH1. PLoS One. 2009; 4: e4434.

5. Radtke C, Redeker J, Jokuszies A, et al. In vivo transformation of neural stem cells following transplantation in the injured nervous system. J Reconstr Microsurg. 2010; 26: 211-212.

6. Siebzehnrubl FA, Jeske I, Müller D, et al. Spontaneous in vitro transformation of adult neural precursors into stem-like cancer cells. Brain Pathol. 2009; 19: 399-408.

7. Tang XQ, Heron P, Mashburn C, et al. Targeting sensory axon regeneration in adult spinal cord. J Neurosci. 2007; 27: 6068-6078.

8. Amariglio N, Hirshberg A, Scheithauer BW, et al. Donor-derived brain tumor following neural stem cell transplantation in an ataxia telangiectasia patient. PLoS Med. 2009; 6: e1000029.

9. Al-Hajj M, Wicha MS, Benito-Hernandez A, et al. Prospective identification of tumorigenic breast cancer cells. Proc Natl Acad Sci U S A 2003; 100: 3983-3988.
10. Hemmati HD, Nakano I, Lazareff JA, et al. Cancerous stem cells can arise from pediatric brain tumors. Proc Natl Acad Sci U S A 2003; 100: 15178-15183.

11. Furnari FB, Fenton T, Bachoo RM, et al. Malignant astrocytic glioma: genetics, biology, and paths to treatment. Genes Dev 2007; 21: 2683-2710.

12. Li X, Xu J, Bai $Y$, et al. Isolation and characterization of neural stem cells from human fetal striatum. Biochem Biophys Res Commun 2005; 326: 425-434.

13. Mapara KY, Stevenson CB, Thompson RC, et al. Stem cells as vehicles for the treatment of brain cancer. Neurosurg Clin $\mathrm{N}$ Am 2007; 18: 71-80.

14. Clarke DL, Johansson CB, Wilbertz J, et al. Generalized potential of adult neural stem cells. Science 2000; 288: 1660-1663.

15. Bjorklund A, Lindvall O. Cell replacement therapies for central nervous system disorders. Nat Neurosci 2000; 3: 537-544.

16. Horner PJ, Gage FH. Regenerating the damaged central nervous system. Nature 2000; 407: 963-970.

17. Lagasse E. Cancer stem cells with genetic instability: the best vehicle with the best engine for cancer. Gene Ther. 2008; 15 : 136-142.

18. Kelly S, Bliss TM, Shah AK, et al. Transplanted human fetal neural stem cells survive, migrate, and differentiate in ischemic rat cerebral cortex. Proc Natl Acad Sci U S A 2004; 101: 11839-11844.

19. Micallef J, Taccone M, Mukherjee J, et al. Epidermal growth factor receptor variant III-induced glioma invasion is mediated through myristoylated alanine-rich protein kinase $C$ substrate overexpression. Cancer Res 2009; 69: 7548-7556.

20. Maher EA, Furnari FB, Bachoo RM, et al. Malignant glioma: genetics and biology of a grave matter. Genes Dev 2001; 15: 1311-1333.

21. Calabrese $\mathrm{C}$, Poppleton $\mathrm{H}$, Kocak M, et al. A perivascular niche for brain tumor stem cells. Cancer Cell 2007; 11: 69-82.

22. Gilbertson RJ, Rich JN. Making a tumour's bed: glioblastoma stem cells and the vascular niche. Nat Rev Cancer 2007; 7: 733-736.

23. Imitola J, Raddassi K, Park KI, et al. Directed migration of neural stem cells to sites of CNS injury by the stromal cell-derived factor 1alpha/CXC chemokine receptor 4 pathway. Proc Natl Acad Sci U S A 2004; 101: 18117-18122.

24. Han $Z$, Ren $H$, Zhao $H$, et al. Hypoxia-inducible factor (HIF)-1\{alpha\} directly enhances the transcriptional activity of stem cell factor (SCF) in response to hypoxia and epidermal growth factor (EGF). Carcinogenesis 2008; 29: 1853-1861.

25. Sun L, Hui A, Su Q, et al. Neuronal and glioma-derived stem cell factor induces angiogenesis within the brain. Cancer Cell 2006; 9: 287-300.

26. Tachibana $\mathrm{K}$, Hirota $\mathrm{S}$, Iizasa $\mathrm{H}$, et al. The chemokine receptor CXCR4 is essential for vascularization of the gastrointestinal tract. Nature 1998; 393: 591-594.

27. Liu Y, Ji R, Li J, et al. Correlation effect of EGFR and CXCR4 and CCR7 chemokine receptors in predicting breast cancer metastasis and prognosis. J Exp Clin Cancer Res 2010; 29: 16.

28. Schlessinger J. Cell signaling by receptor tyrosine kinases. Cell 2000; 103: 211-225.

29. Burger JA, Kipps TJ: CXCR4: a key receptor in the crosstalk between tumor cells and their microenvironment. Blood 2006; 107: 1761-1767.

30. Eck SM, Cote AL, Winkelman WD, et al. CXCR4 and matrix metalloproteinase- 1 are elevated in breast carcinoma-associated fibroblasts and in normal mammary fibroblasts exposed to factors secreted by breast cancer cells. Mol Cancer Res 2009; 7: 1033-1044.

31. Porcile C, Bajetto A, Barbero S, et al. CXCR4 activation induces epidermal growth factor receptor transactivation in an ovarian cancer cell line. Ann N Y Acad Sci 2004; 1030: 162-169. 
32. Schonemeier B, Schulz S, Hoellt V, et al. Enhanced expression of the CXCl12/SDF-1 chemokine receptor CXCR7 after cerebral ischemia in the rat brain. J Neuroimmunol 2008; 198: 39-45.

33. Nicholson RI, Gee JM, Harper ME. EGFR and cancer prognosis. Eur J Cancer 2001; 37 (Suppl 4): S9-15.

34. Venkatakrishnan G, Salgia R, Groopman JE. Chemokine receptors CXCR-1/2 activate mitogen-activated protein kinase via the epidermal growth factor receptor in ovarian cancer cells. J Biol Chem 2000; 275: 6868-6875.

35. Grandal MV, Zandi R, Pedersen MW, et al. EGFRvIII escapes down-regulation due to impaired internalization and sorting to lysosomes. Carcinogenesis 2007; 28: 1408-1417.

36. Rossi G, Marchioni A, Longo L. EGFR mutations and sensitivity to gefitinib. N Engl J Med 2004; 351: 1260-1261.
37. Lee JC, Vivanco I, Beroukhim R, et al. Epidermal growth factor receptor activation in glioblastoma through novel missense mutations in the extracellular domain. PLoS Med 2006; 3: e485.

38. Schwechheimer K, Huang S, Cavenee WK. EGFR gene amplification--rearrangement in human glioblastomas. Int J Cancer 1995; 62: 145-148.

39. Bax DA, Gaspar N, Little SE, et al. EGFRvIII deletion mutations in pediatric high-grade glioma and response to targeted therapy in pediatric glioma cell lines. Clin Cancer Res 2009; 15: 5753-5761.

40. Inda MM, Bonavia R, Mukasa A, et al. Tumor heterogeneity is an active process maintained by a mutant EGFR-induced cytokine circuit in glioblastoma. Genes Dev 2010; 24: 1731-1745.

\section{Additional Figures}

$$
\mathbf{p}<\mathbf{0 . 0 5}
$$

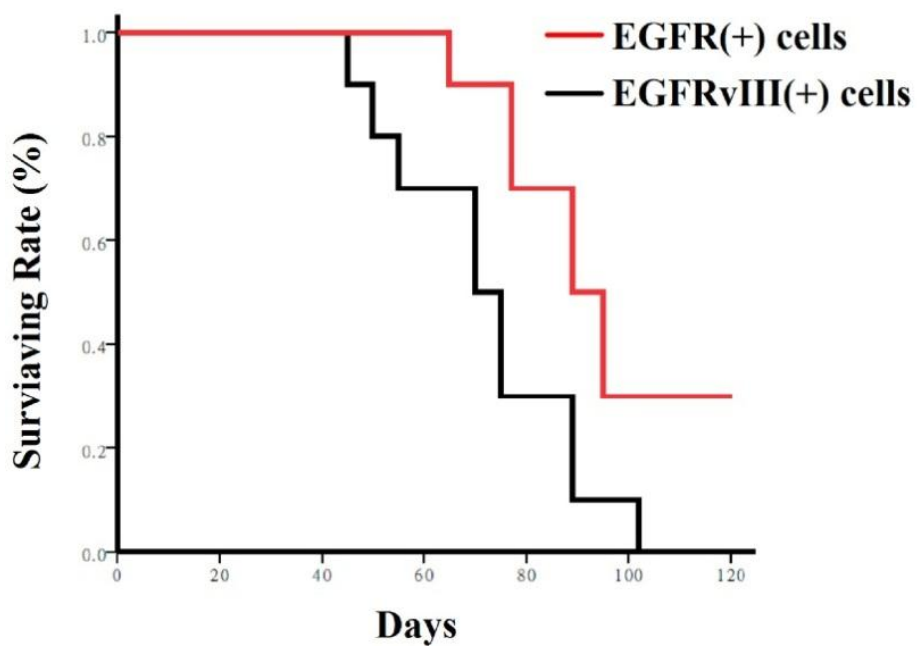

Fig. S1: Nude mice mortality curves. Mice receiving EGFR positive cells lived much longer than the mice receiving EGFRvIII positive cells as shown in Kaplan-Meier survival curves. These cells $\left(10^{4} /\right.$ mouse) were transplanted into the brains of nude mice, respectively. Mice mortality curves were analyzed using the Kaplan-Meier method, with groups compared by respective number of days taken to reach $50 \%$ mortality.
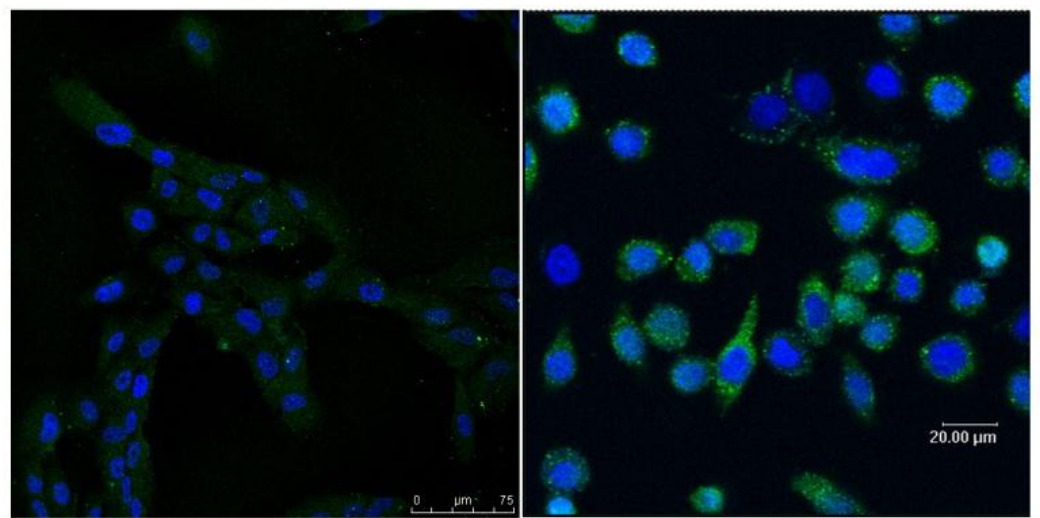

Fig. S2: Immnohistochemistry assay showing different expressed EGFRVIII in hsNSCs (left) and in T1 cells (right). 